Tohoku J. Exp. Med., 2005, 205, 19-25

\title{
Suprascapular Nerve Block versus Steroid Injection for Non-Specific Shoulder Pain
}

\author{
Mehmet Ali Taskaynatan, Bilge Yilmaz, ${ }^{1}$ Ahmet Ozgul, Kamil Yazicioglu ${ }^{1}$ and \\ Tunc Alp Kalyon \\ Department of Physical Medicine and Rehabilitation, and ${ }^{1}$ Turkish Armed \\ Forces Rehabilitation Center, Gulhane Military Medical Academy, Ankara, \\ Turkey
}

Taskaynatan, M.A., Yilmaz, B., Ozgul, A., Yazicioglu, K. and Kalyon, T.A. Suprascapular Nerve Block versus Steroid Injection for Non-Specific Shoulder Pain. Tohoku J. Exp. Med., 2005, 205 (1), 19-25 — Shoulder pain is a common complaint in elder population and may cause an important functional disability. The aim of this study was to compare the effects of suprascapular nerve block with those of steroid injection in patients with non-specific shoulder pain. A total of 60 patients with shoulder pain lasting for more than four weeks were included in this study. After 1:1 randomization, patients were included in the steroid group $(n=30)$ or the suprascapular nerve block group $(n=30)$. Single injection was performed in both groups. All patients were evaluated before treatment, within one week after treatment, and one month later, in terms of pain, range of motion (ROM) (flexion, abduction, internal and external rotations, and total constant shoulder ROM score), satisfaction, and disability (Pennsylvania shoulder scale function score). Steroid injection was applied at two points in order of lateral and anterior routes. Suprascapular nerve block with lidocaine was applied at the suprascapular notch. No difference was noted in the indicated parameters before the treatment between the groups $(p>0.05)$. The difference in all follow-up parameters was statistically significant in the assessment periods in both groups $(p<0.05)$. No method was found to be superior to each other $(p>0.05)$. No complications occurred in suprascapular nerve block in contrary to steroid injection. We suggest that suprascapular nerve block can be considered the preferred treatment for non-specific shoulder pain because of being as effective as steroid injection with rare side effects. - shoulder pain; corticosteroid injection; suprascapular nerve block

(C) 2005 Tohoku University Medical Press

Shoulder pain is a common complaint in elder population that may cause an important functional disability. Prevalence of shoulder pain with disability in general population is approximately 20\% (Philadelphia Panel 2001).
Although there are still many treatment modalities (e.g. non-steroid anti-inflammatory drugs, physical medicine, intra-articular steroid injection, nerve blockade, manipulation and some combinations of these) aiming at increasing range

Received August 30, 2004; revision accepted for publication October 27, 2004.

Address for reprints: Dr. Mehmet Ali Taskaynatan, Department of Physical Medicine and Rehabilitation, Gulhane Military Medical Academy, 06018 Etlik, Ankara, Turkey.

e-mail:matask@gata.edu.tr 
of motion (ROM), relieving pain and as a result improving disability, the results reported about their effectiveness are inconsistent (Green et al. 1998; Philadelphia Panel 2001).

Corticosteroid injection can be used in various shoulder pathologies, primarily in impingement syndrome. In rotator cuff tendon pathologies if adequate progress has not yet been achieved after 4-6 weeks monitoring, a subacromial injection may be considered, especially for patients in whom pain is limiting the ability to perform exercises (Arroyo and Flatow 1999). The suprascapular nerve block may also be useful as part of a comprehensive rehabilitative program for the management of chronic and acute shoulder pain. Its rationale is to block the suprascapular nerve which innervates the $70 \%$ of the shoulder sensory, at suprascapular notch (Ferrante 1999).

The aim of this study was to compare the effects of suprascapular nerve block (SSNB) with those of steroid injection (SI) in non-specific shoulder pain.

\section{Materials ANd Methods}

A total of 60 patients with shoulder pain lasting for more than four weeks were included in the study. After a detailed physical examination, plain shoulder films, routine laboratory tests including complete blood count, sedimentation rate, CRP, brief routine biochemistry, and if necessary RF were ordered for all patients. Soft tissue shoulder ultrasonography (USG) was performed for all patients by a medical doctor who was experienced in soft tissue USG.

The patients with degenerative shoulder pathology in X-rays, and those who were known to have cervical disc pathology, systemic rheumatism, malignancy, stroke, polyneuropathy, carpal tunnel syndrome, mental problems, contraindication to steroid injection (e.g. diabetes mellitus, advanced osteoporosis), known lidocaine drug allergy, deformities of the joints in the affected upper limb, and trauma history in previous 4 weeks were excluded.

After 1:1 randomization, 30 patients were included in the SI group, and 30 patients were included in the SSNB group. All patients were assessed within 5-7 days following the intervention. Subjects were reassessed after one month. A physiatrist who was blinded to the treatment method performed all assessments. The study was approved by our Institutional Ethics Committee and informed consent obtained from each patient.

\section{Assessment scales}

Constant and Pennsylvania shoulder scales are being used widespread in the follow-up of non-specific shoulder pathologies (Leggin and Lannotti 1999). The Constant shoulder score is a 100-point scoring system in which 35 points are derived from the patient's report of pain and function. The remaining 65 points are allocated for objective assessment of range of motion (ROM) and strength (Leggin and Lannotti 1999). Pennsylvania shoulder score that consists of two 100-point scoring systems has been developed in light of deficiencies of available shoulder scoring systems. The self-assessment 100 -point scoring system is based on scoring of the patient's report of pain, satisfaction, and function. The 100-point impairment score consists of objective measures of ROM and strength (Leggin and Lannotti 1999).

\section{Outcome assessment}

Pain. Pain with arm at rest by the side, pain with normal activities, and pain with strenuous activities were assessed with 10-point numeric rating scale with end points of "no pain" (0) and "worst possible pain" (10). Presence of night pain was asked as "yes" or "no". Rest pain and night pain were assessed before and after treatment, and at follow up but pain with normal and strenuous activities were assessed only before treatment and at follow up. Besides getting a score for each item, a total pain score was obtained by subtracting the sum of three pain item scores from 30 .

ROM. Flexion, abduction, external rotation of the painful shoulder were measured both with goniometry and as in Constant shoulder scale. Internal rotation was not measured with goniometry. It was scored as in Constant shoulder scale. ROM measures were obtained before and after the treatment and one month later.

Satisfaction. The patient's satisfaction with the function of the shoulder was assessed with a numeric rating scale with end points of "not satisfied" and "very satisfied." Scoring was based on the number circled by the patient.

Disability. Disability was assessed by using the function part of Pennsylvania shoulder scale. It is based on a 20 -item questionnaire with a four-category Likert scale for responses with maximum 60 points. Scoring of this section was in the following way: 3 points were awarded if the patient "can perform the activity without 
difficulty," 2 points for "some difficulty," 1 point for "much difficulty," and 0 for "can not do at all." Interventions: Two physiatrists, experienced in intra-articular injection methods, who were blinded to the assessment and USG results, performed all injections. Results and complications were recorded. All subjects were taught a simple home exercise program including Codman, strengthening and ROM exercises in pain range.

Steroid injection. All injections were performed at two points in order of lateral and anterior routes. Methylprednisolone acetate of $40 \mathrm{mg}$ ( $1 \mathrm{ml}$ depo-medrol) and $6 \mathrm{ml}$ of $1 \%$ lidocaine (Citanest) were used for this injection. After supporting necessary sterility measures, $2 \mathrm{ml}$ of combined solution was delivered to bisipital sulcus by using anterior route and $5 \mathrm{ml}$ to subacromial space by using lateral route.

SSNB. SSNB was done by using surface anatomy. With the patient seated, the spine of the scapula was identified. A perpendicular line was drawn from the angle of the scapula upward to bisect the spine of the scapula. After supporting necessary sterility measures, $1 \mathrm{ml}$ of $1 \%$ lidocaine was injected subcutaneously for local analgesia, and then $10 \mathrm{ml}$ of lidocaine was delivered into the suprascapular notch after the needle was inserted at the point about $2 \mathrm{~cm}$ lateral to the intersecting point of drawn lines as described in literature (Adriani 1967; Karatas and Meray 2002). Steroid was not involved in the injection solution.

\section{Statistical analysis}

Statistical analysis was performed by the Statistical Package for Social Sciences (SPSS) program version 11.0 for windows (SPSS Inc., Chicago, IL, USA). Demographics were compared by using the Student $t$-test. The results were compared by using the multivariate analysis of variance, and post hoc comparisons were made by using the Mann-Whitney's for differences between the groups and the $t$-test for comparisons of the data within the groups. The relation of demographic data with shoulder symptoms was analyzed using linear regression test. A $p$ value of $<0.05$ was considered to be significant.

\section{RESULTS}

Sixty patients (37 females and 23 males) with the mean age of $57.2 \pm 10.9$ years were included in this study. In SI group, there were 19 females and 11 males with the mean age of $53.8 \pm$
12.1 years (ranged between 31-74 years). A minor trauma by 5 patients $(16.6 \%)$ and overuse by 4 patients $(13.3 \%)$ were declared as a causative event. Duration of the symptoms was 1-3 months in 7 patients $(23.3 \%), 3-6$ months in 7 patients $(23.3 \%)$ and more than 6 months in 16 patients $(53.3 \%)$. Seventeen patients $(56.6 \%)$ had dominant shoulder involvement (Table 1).

In SSNB group, there were 18 females and 12 males with the mean age of $59.9 \pm 9.2$ years (ranged between $40-75$ years). History of minor trauma was present in $4(13.3 \%)$ and overuse in 3 $(10 \%)$ of the patients. Duration of the symptoms was 1-3 months in 7 patients (23.3\%), 3-6 months in 9 patients $(30 \%)$ and more than 6 months in 14 patients $(46.7 \%)$. 19 patients $(63.3 \%)$ had dominant shoulder involvement (Table 1). Two female patients (one in SI group and one in SSNB group) did not come for follow-up assessment.

No complications occurred in SSNB group. Seven patients in SI group complained for increase in pain especially at anterior side. Four patients claimed that the pain disappeared within 3-4 days, but other three patients were still in discomfort at the post treatment assessment. Two patients in SI group claimed that they had suffered from hypertension after the injection, but we could not determine it in any assessment period. At final assessment no patient claimed for any side effect of the interventions.

There were no significant differences between the groups in terms of demographic variables, pain, satisfaction, ROM and disability scores at baseline $(p>0.05)$. The relations of the demographic data with shoulder symptoms were not statistically significant $(p>0.05)$ (Table 1).

After the treatment, all follow-up parameters were significantly improved in both SI and SSNB groups (Tables 2 and 3). The improvements in these parameters obtained from both groups were still significant at the final assessments when compared to the baseline scores (Tables 2 and 3).

No statistically significant difference in the improvements of follow-up parameters was obtained between the groups after the treatment and at the final assessment (Table 4). 
M.A. Taskaynatan et al.

TABLE 1. Demographic data of the patients and their relation with the symptoms

\begin{tabular}{lccc}
\hline & SI Group & SSNB group & Relation $(p)$ \\
\hline Age & $53.75 \pm 12.03$ & $59.93 \pm 9.14$ & 0.277 \\
Sex (female/male) & $19(63.3 \%) / 11(36.7 \%)$ & $18(60 \%) / 12(40 \%)$ & 0.323 \\
Dominant shoulder & $17(56.6 \%)$ & $19(63.3 \%)$ & 0.635 \\
Sleep position* & & & 0.314 \\
Affected side & $4(13.3 \%)$ & $8(26.7 \%)$ & \\
Other side & $8(26.7 \%)$ & $5(16.7 \%)$ & \\
Various & $18(60 \%)$ & $17(56.7 \%)$ & 0.541 \\
Precipitant factor & & & \\
Overuse & $4(13.3 \%)$ & $3(10 \%)$ & 0.344 \\
Minor trauma & $5(16.6 \%)$ & $4(13.3 \%)$ & \\
Symptom duration & $7(23.3 \%)$ & $7(23.3 \%)$ & \\
1-3 months & $7(23.3 \%)$ & $9(30 \%)$ & \\
3-6 months & $16(53.3 \%)$ & $14(46.7 \%)$ & \\
$>6$ months & &
\end{tabular}

* Before shoulder pathology.

TABLE 2. Differences in follow-up parameters in the suprascapular nerve block group

\begin{tabular}{|c|c|c|c|c|c|}
\hline & \multirow{2}{*}{$\begin{array}{c}\begin{array}{c}\text { Before } \\
\text { treatment }\end{array} \\
\text { Mean } \pm \text { S.D. }\end{array}$} & \multicolumn{2}{|c|}{ After treatment } & \multicolumn{2}{|c|}{ Final assessment } \\
\hline & & Mean \pm S.D. & $p^{*}$ & Mean \pm S.D. & $p^{*}$ \\
\hline Rest pain & $3.1 \pm 2.1$ & $2.3 \pm 1.4$ & 0.007 & $2.5 \pm 1.4$ & 0.03 \\
\hline Pain with normal activity & $5.4 \pm 1.5$ & - & - & $4.8 \pm 1.4$ & 0.024 \\
\hline Pain with strenuous activities & $7.7 \pm 1.2$ & - & - & $7.2 \pm 1.2$ & 0.047 \\
\hline Satisfaction & $4 \pm 1.6$ & - & - & $4.6 \pm 1.6$ & 0.016 \\
\hline Flexion & $126 \pm 32$ & $134 \pm 29$ & 0.012 & $136 \pm 32$ & 0.014 \\
\hline Abduction & $119 \pm 38$ & $125 \pm 37$ & 0.014 & $126 \pm 38$ & 0.037 \\
\hline External rotation & $74 \pm 11$ & $77 \pm 11$ & 0.016 & $76 \pm 11$ & 0.035 \\
\hline Constant internal rotation & $5.4 \pm 2.8$ & $5.9 \pm 2.1$ & 0.009 & $5.87 \pm 2.5$ & 0.032 \\
\hline Constant total ROM & $26.2 \pm 8.1$ & $28 \pm 7.6$ & 0.005 & $27.9 \pm 8$ & 0.014 \\
\hline Pennsylvania total pain & $13.7 \pm 4.3$ & - & - & $15.6 \pm 3$ & 0.013 \\
\hline Pennsylvania function & $23.2 \pm 7.6$ & - & - & $25.7 \pm 9.2$ & 0.023 \\
\hline
\end{tabular}

S.D., standard deviation; ROM, range of motion.

*According to the baseline values. 
TABLE 3. Differences in follow-up parameters in the steroid injection group

\begin{tabular}{|c|c|c|c|c|c|}
\hline & \multirow{2}{*}{$\begin{array}{c}\begin{array}{c}\text { Before } \\
\text { treatment }\end{array} \\
\text { Mean } \pm \text { S.D. }\end{array}$} & \multicolumn{2}{|c|}{ After treatment } & \multicolumn{2}{|c|}{ Final assessment } \\
\hline & & Mean \pm S.D. & $p^{*}$ & Mean \pm S.D. & $p^{*}$ \\
\hline Rest pain & $2.9 \pm 1.9$ & $2.1 \pm 1.1$ & 0.004 & $2.5 \pm 1.3$ & 0.037 \\
\hline Pain with normal activity & $5.7 \pm 1.6$ & - & - & $5.1 \pm 1.2$ & 0.036 \\
\hline Pain with strenuous activities & $7.8 \pm 1.2$ & - & - & $7.3 \pm 0.9$ & 0.050 \\
\hline Satisfaction & $3.9 \pm 1.4$ & - & - & $4.9 \pm 1.8$ & 0.008 \\
\hline Flexion & $130 \pm 39$ & $148 \pm 33$ & 0.008 & $143 \pm 37$ & 0.014 \\
\hline Abduction & $120 \pm 43$ & $134 \pm 44$ & 0.022 & $132 \pm 42$ & 0.017 \\
\hline External rotation & $76 \pm 10$ & $79 \pm 10$ & 0.013 & $79 \pm 9$ & 0.046 \\
\hline Constant internal rotation & $5.1 \pm 3.1$ & $5.8 \pm 3.3$ & 0.029 & $5.4 \pm 3.2$ & 0.043 \\
\hline Constant total ROM & $25.4 \pm 9.6$ & $28.6 \pm 9.5$ & 0.009 & $27.5 \pm 9.1$ & 0.012 \\
\hline Pennsylvania total pain & $13.5 \pm 4$ & - & - & $15.2 \pm 2.3$ & 0.01 \\
\hline Pennsylvania function & $24.2 \pm 7.4$ & - & - & $28.9 \pm 9.9$ & 0.009 \\
\hline
\end{tabular}

S.D., standard deviation; ROM, range of motion.

*According to the baseline values.

TABLE 4. Differences of improvements between the groups

\begin{tabular}{lccccc}
\hline & \multicolumn{2}{c}{ After treatment } & & \multicolumn{2}{c}{ Final assessment } \\
\cline { 2 - 5 } \cline { 5 - 5 } & \multicolumn{1}{c}{$t$} & $p$ & $t$ & 0.57 \\
\hline Night pain & -0.182 & 0.67 & & -0.283 & 0.57 \\
Rest pain & 0.021 & 0.984 & & 0.373 & 0.711 \\
Pain with normal activity & - & - & 0.105 & 0.917 \\
Pain with strenuous activities & - & - & -0.023 & 0.982 \\
Satisfaction & - & -0.938 & 0.353 \\
Flexion & 1.045 & 0.303 & & -0.440 & 0.662 \\
Abduction & 0.343 & 0.734 & & -1.199 & 0.239 \\
External rotation & 0.181 & 0.857 & & -0.086 & 0.932 \\
Constant internal rotation & -0.019 & 0.985 & & -0.941 & 0.352 \\
Constant total ROM & 0.746 & 0.460 & & 0.435 & 0.666 \\
Pennsylvania total pain & - & - & 0.230 & 0.819 \\
Pennsylvania function & - & - & -1.013 & 0.317 \\
\hline
\end{tabular}

ROM, range of motion.

\section{Discussion}

It is not always easy to find the origin of the pain in shoulder because of its rather complex anatomy. Although there are many specific tests for differential diagnosis of the shoulder pathologies, not much data on their diagnostic value are available (Naredo et al. 2002). As a result especially in outpatient services, physicians mostly face a patient with a shoulder pain of unknown origin. SI and SSNB are two therapeutic interventions that can be tried in patients that shoulder pain does not resolve despite medical treatment and rest. 
SI was found to be effective in non-specific shoulder pain in many studies (Adebajo et al. 1990; Eustace et al. 1997; Green et al. 1998; van der Windt et al. 1998). There are comparatively fewer studies of SSNB than SI. It has been studied in glenohumeral arthritis (Brown et al. 1988), frozen shoulder (Karatas and Meray 2002), rheumatoid arthritis and osteoarthritis (Gado and Emery 1993; Shanahan et al. 2003), hemiplegic shoulder (Lee and Khunadorn 1986), rotator cuff lesions (Vecchio et al. 1993) and found to be effective in these clinical pictures. Up to our knowledge, we are the first comparing the effects of these two interventions in non-specific shoulder pain.

In literature results of the studies about steroid injection in shoulder pain are controversial. Different steroid preparations have been used and injections have been done by using different routes, and results have usually been compared with physiotherapy (Green et al. 1998). Van der Windt et al. have reported that beneficial effects of steroid injections, administered from posterior route by general practioners, were still active in $77 \%$ of patients at the end of seven weeks (van der Windt et al. 1998). Eustace et al. have suggested that effect of steroid injection is more beneficial and lasts longer if it is administered at proper site (Eustace et al. 1997). Biceps tendon and subacromial bursa pathologies accompany most of the rotator cuff lesions (Arslan et al. 1999), but how much they have role in pain and ROM restriction is not very clear yet (Sethi et al. 1999). In the present study we observed biceps tendon pathology with USG in $69 \%$ of the patients. In light of this knowledge we used anterior route besides lateral route in purpose of being effective on biceps pathologies accompanying other shoulder pathologies. After SI, short-term improvement in ROM restriction has been obtained in all directions in most studies, but some of the authors have suggested that improvement in abduction or rotations has been lost in long-term follow-up (Eustace et al. 1997). In the present study despite only one session steroid injection was applied, a significant improvement that was still present at the final assessment in all follow- up parameters was obtained.

Although it has been found useful as an aid to physical reconditioning, too little data exist in the literature to determine the effectiveness of the SSNB when used alone in the treatment of shoulder conditions. Similarly, few data exist about the optimal frequency for repetition of suprascapular nerve block. The exact mechanism of SSNB is still unknown. Pain relief from the block extends beyond the pharmacological effect of the drug. A decrease in central sensitization of dorsal horn nociceptive neurons or "wind down" theories have been suggested. A decrease in algogenic substances and direct infiltration of the supraspinatus muscle have been suggested as possible contributing factors (Shanahan et al. 2003). Although it generally has been used with steroid combination, there is no consensus about its necessity. Some authors have suggested that delivering the solution into the suprascapular fossa is effective enough for nerve block but Karatas and Meray have found that nerve block close to the nerve with EMG guide is more effective (Karatas and Meray 2002). In the present study only lidocaine was delivered into the suprascapular notch, so close to the nerve, and a significant improvement that was still present at the final assessment in all follow-up parameters was obtained.

There is no worldwide accepted clinical classification on shoulder problems. Although in the present study all patients were evaluated with USG at baseline, we did not intend to classify the patients based on the USG data because of its own limitations and to prevent confusing consequences in expressing and understanding of the results. We therefore compared the effects of the SI and SSNB methods, on non-specific shoulder pain that is encountered frequently in outpatient services.

In summaly, both SI and SSNB have similar efficacies for non-specific shoulder pain. Because there are more contraindications to steroid, undesirable results may occur if SI is applied without radiological confirmation of shoulder pathology. SSNB may be prefered treatment for non-specific shoulder pain. 


\section{References}

Adebajo, A., Nash, P. \& Hazleman, B.L. (1990) A prospective double blind dummy controlled study comparing triamcinolone hexacetodine injection with oral diclofenac $50 \mathrm{mg}$ tds in patients with rotator cuff tendinitis. J. Rheumatol., 17, 1207-1210.

Adriani, J. (1967) Labat's Regional Anesthesia. 3rd edition, WB Saunders, Philadelphia.

Arroyo, J.S. \& Flatow, E.L. (1999) Management of Rotator Cuff Disease: Intact and Repairable Cuff. In: Disorders of the Shoulder: Diagnosis and Treatment, Edited by J.P. Lannotti and G.R. Williams, Lippincott, Williams \& Wilkins, Philadelphia, pp. 31-56.

Arslan, G., Apaydin, A., Kabaalioglu, A., Sindel, T. \& Luleci, E. (1999) Sonographically detected subacromial/subdeltoid bursal effusion and biceps tendon sheath fluid: reliable signs of rotator cuff tear. J. Clin. Ultrasound, 27, 335-339.

Brown, D.E., James, D.C. \& Roy, S. (1988) Pain relief by suprascapular nerve block in glenohumeral artritis. Scan. J. Rheumatol., 17, 411-415.

Eustace, J.A., Brophy, D.P., Gibney, R.P., Bresnihan, B. \& FitzGerald, O. (1997) Comparison of the accuracy of steroid placement with clinical outcome in patients with shoulder symptoms. Ann. Rheum. Dis., 56, 59-63.

Ferrante, F.M. (1999) Medical management of chronic shoulder pain. In: Disorders of the Shoulder: Diagnosis and Treatment, Edited by J.P. Lannotti and G.R. Williams, Lippincott, Williams \& Wilkins, Philadelphia, pp. 1043-1074.

Gado, K. \& Emery, P. (1993) Modified suprascapular nerve block with bupivacaine alone effectively controls chronic shoulder pain in patients with rheumotoid arthritis. Ann. Rheum. Dis., 52, 215-218.

Green, S., Buchbinder, R., Glazier, R. \& Forbes, A. (1998) Systematic review of randomized controlled trials of interventions for painful shoul- der: selection criteria, outcome assessment, and efficacy. BMJ., 316, 354-360.

Karatas, G.K. \& Meray, J. (2002) Suprascapular nerve block for pain relief in adhesive capsulitis: Comparison of 2 different techniques. Arch. Phys. Med. Rehabil., 83, 593-597.

Lee, K. \& Khunadorn, F. (1986) Painful shoulder in hemiplegic patients: a study of the suprascapular nerve. Arch. Phys. Med. Rehabil., 67, 818-820.

Leggin, B.G. \& Lannotti, J.P. (1999) Shoulder outcomes. In: Disorders of the Shoulder: Diagnosis and Treatment, Edited by J.P. Lannotti and G.R. Williams, Lippincott, Williams \& Wilkins, Philadelphia, pp. 1023-1042.

Naredo, E., Aguado, P., De Miguel, E., Uson, J., Mayordomo, L., Gijon-Banos, J. \& MartinMola, E. (2002) Painful shoulder: Comparison of physical examination and ultrasonographic findings. Ann. Rheum. Dis., 61, 132-136.

Philadelphia Panel. (2001) Philadelphia panel evidence-based clinical practice guidelines on selected rehabilitation interventions for shoulder pain. Phys. Ther., 81, 1719-1730.

Sethi, N., Wright, R. \& Yamaguchi, K. (1999) Disorders of the long head of the biceps tendon. $J$. Shoulder. Elbow. Surg., 8, 644-654.

Shanahan, E.M., Ahern, M., Smith, M., Wetherall, M., Bresnihan, B. \& FitzGerald, O. (2003) Suprascapular nerve block (using bupivacaine and methylprednisolone acetate) in chronic shoulder pain. Ann. Rheum. Dis., 62, 400-406.

van der Windt, D.A., Koes, B.W., Deville, W., Boeke, A.J., de Jong, B.A. \& Bouter, L.M. (1998) Effectiveness of corticosteroid injections versus physiotherapy for treatment of painful stiff shoulder in primary care: randomized trial. BMJ., 317, 1292-1296.

Vecchio, P.C., Adebajo, A.O. \& Hazleman, B.L. (1993) Suprascapular nerve block for persistent rotator cuff lesions. J. Rheumatol., 20, 453-455. 